\title{
Las dimensiones teóricas del clúster y su aplicación al turismo médico
}

\author{
As dimensões teóricas do cluster e seu aplicativo ao turismo médico \\ Cluster's theoretical dimensions and its application to medical tourism
}

\author{
Jorge Carlos Morgan Medina ${ }^{1}$ \\ Onésimo Cuamea Velázquez ${ }^{1}$ \\ Ario Refugio Estrada Gaxiola ${ }^{1}$
}

Recibido el: 16/11/2018. Revisado e aprobado el: 19/06/2019. Aceptado el: 25/07/2019. DOI: http://dx.doi.org/10.20435/inter.v21i3.2251

Resumen: Porter tiene dos versiones del concepto de clúster. En la primera versión, de 1990, afirma que, a nivel de la economía de un país, la industria es el elemento básico. Las interrelaciones claves son entre industrias, ya sean de tipo horizontal o vertical. En la segunda versión, del año 2000, sostiene que, a nivel de un área local o región, las firmas y las interrelaciones entre ellas son el elemento clave. En esta segunda versión, la ubicación geográfica y los factores socioculturales son exógenos a su generación y desarrollo. En consecuencia, las particularidades territoriales y las socio-culturales dentro del territorio no determinan los clústeres de empresas o la generación de las ventajas competitivas. La aplicación de esta metodología al turismo es una tarea reciente, incluso la investigación de este enfoque aplicada al sector de servicios, especialmente en el turismo médico, es un área nueva y, por lo tanto, poco desarrollada. De alguna manera, a este tipo de agrupaciones se les han aplicado los mismos principios que a los clústeres industriales. Una diferencia fundamental entre cualquier clúster industrial y un clúster de turismo estriba en el hecho que, en el primero, es el producto el que se desplaza fuera del lugar de producción hasta el lugar de consumo; mientras en cualquier clúster turístico sucede todo lo contrario, es decir, la demanda (el turista) es lo que debe trasladarse hacia el lugar de producción del producto turístico. Esta diferencia esencial repercute directamente en la manera de planear y gestionar un clúster de turismo médico.

Palabras clave: clúster; turismo médico; clusterización; ventaja competitiva.

Resumo: Porter tem duas versões do conceito de cluster. Na primeira versão, de 1990, afirma que, em nível da economia, a indústria de um país é o elemento básico. As inter-relações-chave são entre indústrias, sejam o tipo horizontal ou vertical. Na segunda versão, de 2000, argumenta que, em nível de uma área local ou região, as empresas e as inter-relações entre elas são o elemento-chave. Nesta segunda versão, a localização geográfica e os fatores socioculturais são exógenos a sua geração e desenvolvimento. Consequentemente, as especificidades territoriais e socioculturais no território não determinam os clusters de empresas ou a geração das vantagens competitivas. A aplicação dessa metodologia para o turismo é uma tarefa recente, até mesmo a pesquisa dessa abordagem aplicada ao setor de serviços, especialmente no turismo médico, é uma área nova e, portanto, subdesenvolvida. De alguma forma, esses tipos de grupos têm aplicado os mesmos princípios para clusters industriais. Uma diferença fundamental entre um cluster industrial e um cluster de turismo reside no fato de que o primeiro é o produto que viaja para fora do local de produção até o local de consumo, enquanto em qualquer cluster turístico acontece o contrário, isto é, a demanda (os turistas) é que deve ir para o local de produção do produto turístico. Esta diferença essencial afeta diretamente a maneira de planejar e gerenciar um cluster de turismo médico.

Palavras-chave: cluster; turismo médico; clusterização; vantagem competitiva.

Abstract: Porter has two versions of the cluster concept. In 1990, the first version says that, in terms of the economy of a country, "industry" is the basic element. The key relationships are between industries, whether horizontal or vertical. In the second version, from 2000, he argues that the level of a local area or region firms and interrelations between them are the key element. In this second version, geographic location and socio-cultural factors are exogenous to his generation and development. Consequently, territorial specificities and within the socio-cultural territory not determine clusters of companies or the generation of competitive advantages. The application of this methodology to tourism is a recent task, even investigating this approach applied to the service sector, especially in the medical tourism, is a new area and, therefore, underdeveloped. Somehow, this type of groups has been applied the same principles to industrial clusters. A fundamental difference between any industrial cluster and tourism cluster lies in the fact that the former is the product that moves beyond the place of production to the place of consumption; as in any tourist cluster, the opposite

\footnotetext{
${ }^{1}$ Universidad Autónoma de Baja California (UABC), México.
} 
happens, the demand side (tourists) is what should move to the place of production of the tourism product. This essential difference directly affects the way to plan and manage a cluster of medical tourism.

Keywords: cluster; medical tourism; clustering; competitive advantage.

\section{INTRODUCCIÓN}

Si se considera que el turismo de masas a escala internacional (global) es un fenómeno relativamente reciente, que empezó a cobrar importancia a mediados del Siglo XX (1950), y que la teoría de clúster se incorporó a esta actividad a finales del mismo siglo (1990), entonces se tiene un escenario relativamente nuevo en el que muchos de sus problemas, procesos, manifestaciones y efectos se encuentran en una fase de 'experimentación' o adaptación. Para el propósito de este trabajo, se decidió incluir solamente aspectos relacionados a la teoría aplicada a la actividad turística, haciendo énfasis en los de turismo médico.

Turner (2007), señala que la frase turismo de bienestar, se utiliza para caracterizar a aquellos viajeros que visitan Spas, clínicas de masajes terapéuticos, retiros espirituales o centros donde se practican terapias para el rejuvenecimiento; en el mismo sentido, el turismo de salud, se usa para designar a quienes recurren a la medicina preventiva; y por último, el turismo médico, se refiere a aquellos viajeros que acuden a realizarse algún tratamiento quirúrgico, incluso tratamientos de fertilidad.

Aplicar el enfoque de clúster para analizar el turismo médico, requiere incorporar un primer aspecto que consiste en evaluar si este enfoque, desarrollado principalmente para explicar actividades industriales de exportación, resulta válido para aplicarlo a este sector. En este sentido, Castillo y Lozano (2006), sostienen que desde esta perspectiva el turismo demanda una estrategia de investigación que abone al esfuerzo por el análisis crítico reflexivo a partir de concebir la importancia del problema epistemológico en la construcción de su conocimiento; la identificación de condiciones esenciales que caractericen los fenómenos en cuestión; e identificar los elementos básicos sobre los que se deba intervenir para dirigir y operar sus múltiples manifestaciones.

Como consecuencia, Una conceptualización deficiente o parcial de clúster de turismo médico impide una comprensión holística del fenómeno, por lo que incide negativamente en su desarrollo competitivo, y en la falta de métodos, técnicas y procedimientos que permitan una medición y análisis integral del fenómeno y sus complejas manifestaciones.

En este sentido, este estudio tiene como objetivo analizar la pertinencia de la aplicación del enfoque teórico-conceptual del de clúster y su aplicación al segmento de turismo médico, y contribuir al escaso conocimiento sobre los retos teóricos-conceptuales que determinarían su correcta aplicación.

\section{ORÍGENES, CONCEPTUALIZACIÓN Y APLICACIÓN DEL ENFOQUE DE CLÚSTER}

Con el propósito de promover el desarrollo económico a nivel local, en la última década se ha impulsado la implementación de este tipo de clústeres, basados en el diamante de competitividad de Michael Porter (1998), quien afirma que son concentraciones geográficas de empresas e instituciones interconectadas en un campo particular, y que incluyen a una variedad de industrias y entidades orientadas a la competencia. 
Figura 1 - Diamante de competitividad de Porter

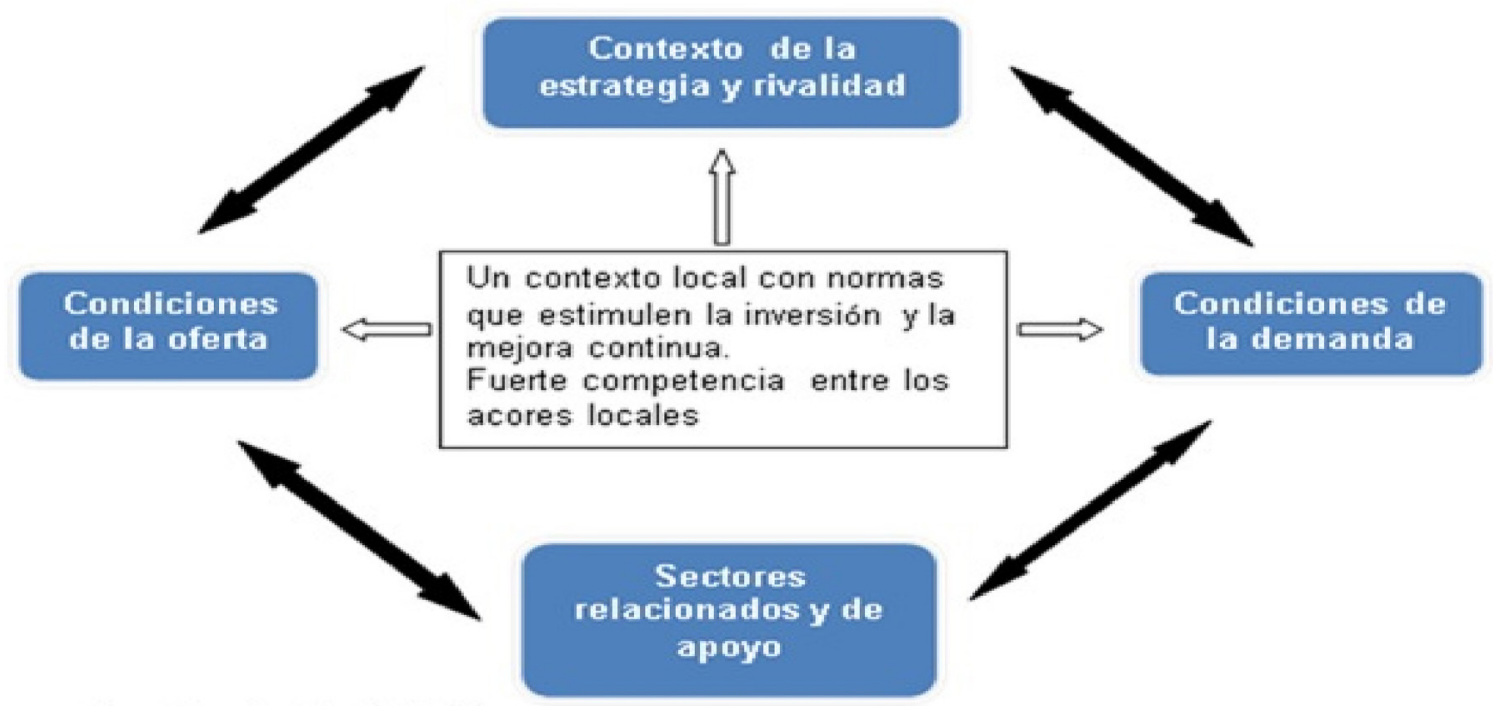

Fuente: Porter (2001).

De acuerdo con Tello (2008), Porter tiene dos versiones del concepto de clúster. En la primera versión de 1990, a nivel de la economía de un país la 'industria' es el elemento básico. Las interrelaciones claves son entre industrias, ya sean de tipo horizontal o vertical. En la segunda del año 2000, a nivel de un área local o región, las firmas y las interrelaciones entre ellas son el elemento clave. En esta versión, la ubicación geográfica y los factores socioculturales son exógenos a su generación y desarrollo. Continuando con Tello (2008), en consecuencia, las particularidades territoriales y las socio-culturales dentro del territorio no determinan los clústeres de empresas o la generación de las ventajas competitivas.

Lato sensu, todo clúster es un agrupamiento o sistema productivo que tiene un alcance local, lo que no impide que pueda desarrollarse a otros niveles geográficos, ya sea estatal o regional. Sin embargo, es necesario señalar que no existe un solo enfoque dominante para conceptualizarlos, sino que en ellos convergen una serie de perspectivas teóricas que permiten analizarlos con mayor detenimiento desde distintas visiones. Tal como lo afirma Dahl (2001), todos estos nuevos enfoques que tratan de explicar las aglomeraciones geográficas y clusterizaciones, representan una especia de convergencia de los principales postulados de las escuelas teóricas tradicionales ya que incorporan uno o varios de sus elementos. 
Figura 2 - Enfoques que convergen en la teoría económica de los clústeres

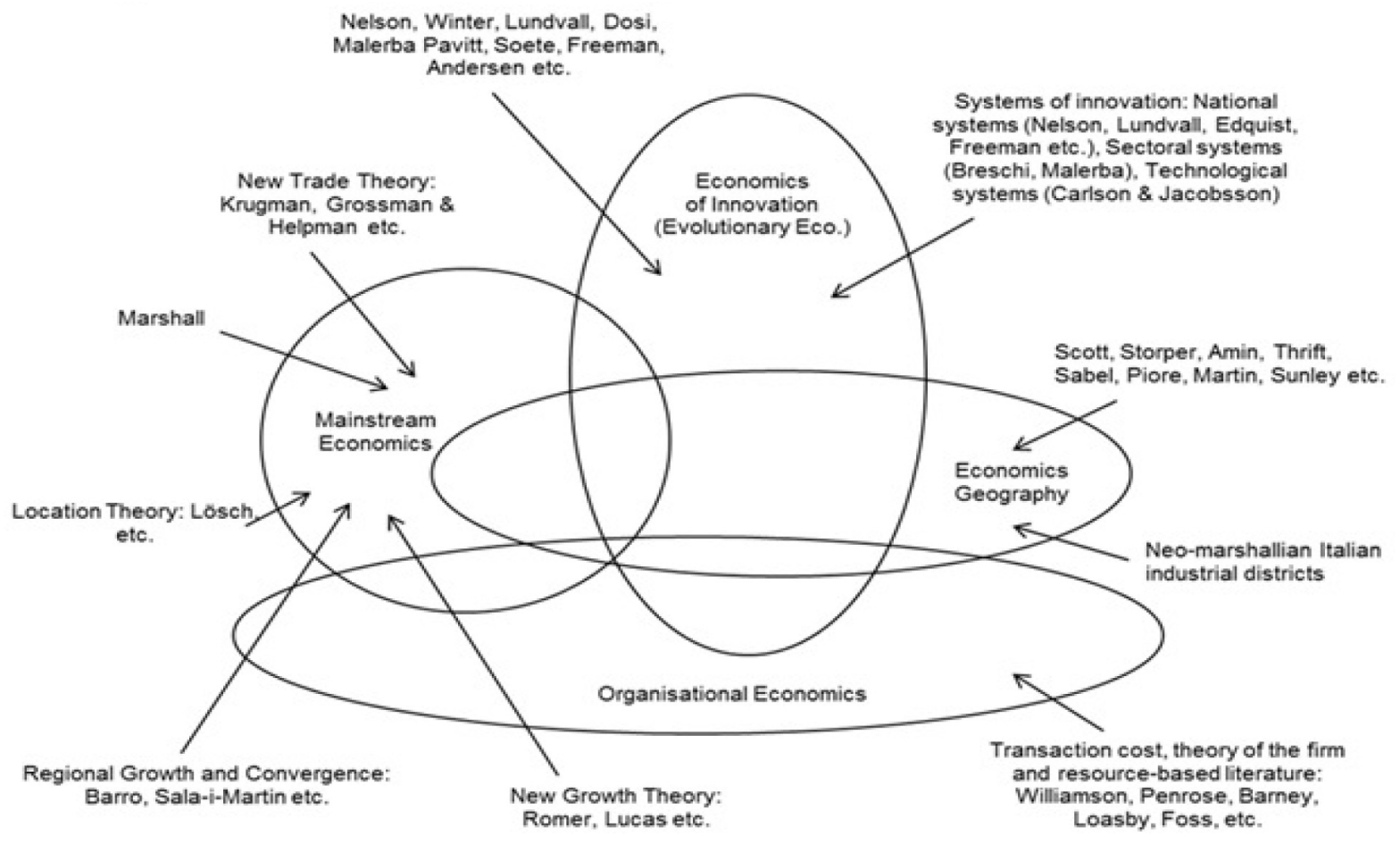

Fuente: Dahl (2001).

Por su parte, al analizar los factores que propician el desarrollo económico local, Tello (2008) afirma que el paso del análisis de la economía nacional al de un área geográfica local dentro de dicha economía está sujeta a discusión, ya que existen cuatro factores adicionales que incorporan las teorías de desarrollo a nivel local con respecto a aquellas a nivel nacional:

1ㅇ) Que las áreas locales no son homogéneas en todo un país, y que esas diferencias son las que explican los diferentes niveles de desarrollo en cada localidad.

2ㅇ) Los agentes o actores privados pueden tener mayor importancia y relevancia - que el Estado - en la dinámica del desarrollo de las áreas geográficas.

3ㅇ) La diferencia que existe entre los bienes y servicios públicos proporcionados por cada orden de gobierno.

4ㅇ) El carácter multidisciplinario en proceso de desarrollo económico local, que se originó como respuesta a las prácticas implementadas por los gobiernos federales o estatales.

Por otro lado, los tipos de dimensiones del análisis que permitirían clasificar los estudios y el concepto de clúster empleado son: 1) tipo de relación (interdependencia o similitud) entre empresas o sectores; 2) tipo de flujos (de productos o de conocimientos); 3) nivel de análisis (micro, meso o macro); 4) límites espaciales del clúster (nacional, regional o local); 5) organizaciones e instituciones tomados en consideración. A pesar de estos esfuerzos, todavía no ofrecen un marco preciso y ordenado que oriente la política industrial (NAVARRO, 2001).

Este enfoque se ha aplicado tanto a nivel nacional, como en regiones, estados o en localidades, pero escasamente se ha incluido al sector turismo en toda su complejidad. Tello (2008) presenta una muestra de clústeres para países desarrollados desde 1971 hasta el año 2002. El estudio presenta dos tipos de agrupamientos, en primer lugar, aquellos que se enfocaron de cadenas productivas internacionales que se desarrollaron a nivel nacional en Estados Unidos, 
Suiza, Suecia, Alemania, Japón, Italia, Reino Unido y Corea del Sur. Todos referidos al sector industrial, destacando la industria del acero y hierro, productos forestales y de madera, químicos y petroquímicos, productos de diversiones, semiconductores y computadoras, entre otros.

Mientras que entre los que se avocaron a la creación de sistemas de innovación se ubican Australia, Austria, Bélgica, Dinamarca, Estados Unidos, Holanda y Suecia. Destacando las actividades de comercio y servicios, construcción, agro-alimentos, puertos, transporte y comunicaciones. En los de nivel regional o en áreas locales, destaca el clúster de casinos y diversiones, ubicado en Las Vegas, Nevada. El resto de los casos mencionados son similares a los descritos en la fase nacional, con sus respectivas variaciones en la dimensión geográfica y de clasificación en el sector industrial, ya que los hay desde la industria automotriz, aeroplanos y helicópteros, joyas, seguros, tele-marketing, reservaciones de hotel, hasta los que se dedican a la producción de binoculares, anteojos, utensilios de cocina, entre otros, como se aprecia, una gama muy variada de productos.

En resumen, casi la totalidad de los clústeres presentados en la literatura, se refieren al sector industrial, a productos de exportación, caracterizados por un aspecto esencial: los productos son desplazados desde el lugar de su producción, hasta los países donde se ubica la demanda que habrá de adquirirlos. Este simple hecho resulta fundamental para la conceptualización y gestión de un clúster de turismo, ya que en este sector el proceso se realiza a la inversa. Es decir, la demanda se traslada hacia el sitio donde se consume el producto turístico. Estas implicaciones conceptuales y operativas se explicarán en el siguiente apartado.

Ketels, Lindkvist y Sölvel (2006) afirman que, en las economías en desarrollo, las iniciativas de clústeres suelen concentrarse en las industrias básicas como la agricultura, muebles y textiles. En las economías avanzadas se orientan preferentemente hacia las industrias de alta tecnología, por ejemplo, la biotecnología. Mientras que en las economías en transición suele haber una mezcla de intereses que se orientan tanto a las industrias básicas como a las de alta tecnología. El turismo, por su parte, a veces resulta de interés para las economías en desarrollo o en transición, pero rara vez en las avanzadas.

Sin importar el nivel del desarrollo del país donde se localicen, Navarro (2003), identifica los aspectos que deben considerarse para el gobierno de los clústeres, señala que en la mayoría de los casos se ha buscado la participación del sector privado. Existen diferentes tipos de organizaciones en las que se pueden conformar, algunas son públicas (Departamento de desarrollo del gobierno local), otras son privadas (Asociaciones sectoriales o cámaras de comercio), y otras son mixtas públicas-privadas. Explica también, que tales organizaciones proporcionan un foro para la colaboración e interacción entre las empresas, son un interfaz entre las empresas y el gobierno, y son el mecanismo que propicia la interaccione entre las empresas y las instituciones de apoyo (Universidades, centros de investigación, instituciones financieras, entre otras), al mismo tiempo que proveen determinados servicios de apoyo. Es decir, las principales funciones que realizan son, funcionar como lobby al coordinar las acciones público-privadas, obtener apoyo para programas especiales. En menor medida llevan a cabo la coordinación de actividades de formación más investigación y desarrollo, y con menor frecuencia las relacionadas con investigación de mercados y marcas conjuntas, compras conjuntas, etc. (NAVARRO, 2003). De ahí la importancia de la formación, preparación y experiencia de quienes lo dirijan, o de alguna manera, participen en alguna de sus fases o procesos, considerando la etapa de desarrollo en la que se encuentre.

Todo clúster pasa por seis etapas diferentes (IRE, 2001): 
1. El nacimiento de un clúster se remonta a circunstancias históricas, tales como la disponibilidad de materias primas.

2. Una vez que una aglomeración de empresas se ha establecido, se crean más externalidades económicas, formando un proceso acumulativo.

3. La formación de nuevas organizaciones que atienden a varias empresas del creciente clúster.

4. El desarrollo de economías externas y el surgimiento de nuevas organizaciones locales aumenta la visibilidad, prestigio y el atractivo de un clúster.

5. La creación del no mercado, los bienes relacionados que fomentan la circulación de la información y el conocimiento, a través de la colaboración informal, facilitan la coordinación de la actividad económica.

6. Aun cuando puede prolongar su éxito por décadas integrarse a uno nuevo, tarde o temprano enfrentara un periodo de declinación o decadencia.

Los clústeres pueden pasar por distintos niveles en su etapa de desarrollo, pero en general, surgen debido a la comercialización de la base científica existente. Brown (2000), afirma que las fases de su ciclo de vida podrían caracterizarse de la siguiente manera:

1ạ Etapa: La plataforma de investigación crea la tecnología que sirve de base para el desarrollo futuro de la agrupación.

2a Etapa: La base de investigación comercializa la tecnología, ya se internamente o por medio de contrato con terceros.

3a Etapa: las empresas empiezan a crecer y desarrollarse en torno a la tecnología, creando un pequeño grupo de empresas interrelacionadas. Los niveles de empleo siguen siendo muy bajos.

4a Etapa: Los servicios especializados de apoyo y proveedores emergen para dar servicio a los integrantes, añadiendo profundidad y capacidad innovadora a todos los integrantes del clúster.

5a Etapa: Las empresas empiezan a crecer y a expandir sus capacidades. A menudo, los organismos de comercio y las redes informales crecen durante esta etapa.

6a Etapa: Las empresas empiezan a alcanzar cierto grado de madurez, y podrían tener que empezar a diversificarse para continuar en el negocio. Puede ser el momento de actualizar los vínculos con las universidades, centros de investigación u otros proveedores de tecnología.

Figura 3 - Etapas del ciclo de vida de una aglomeración productiva

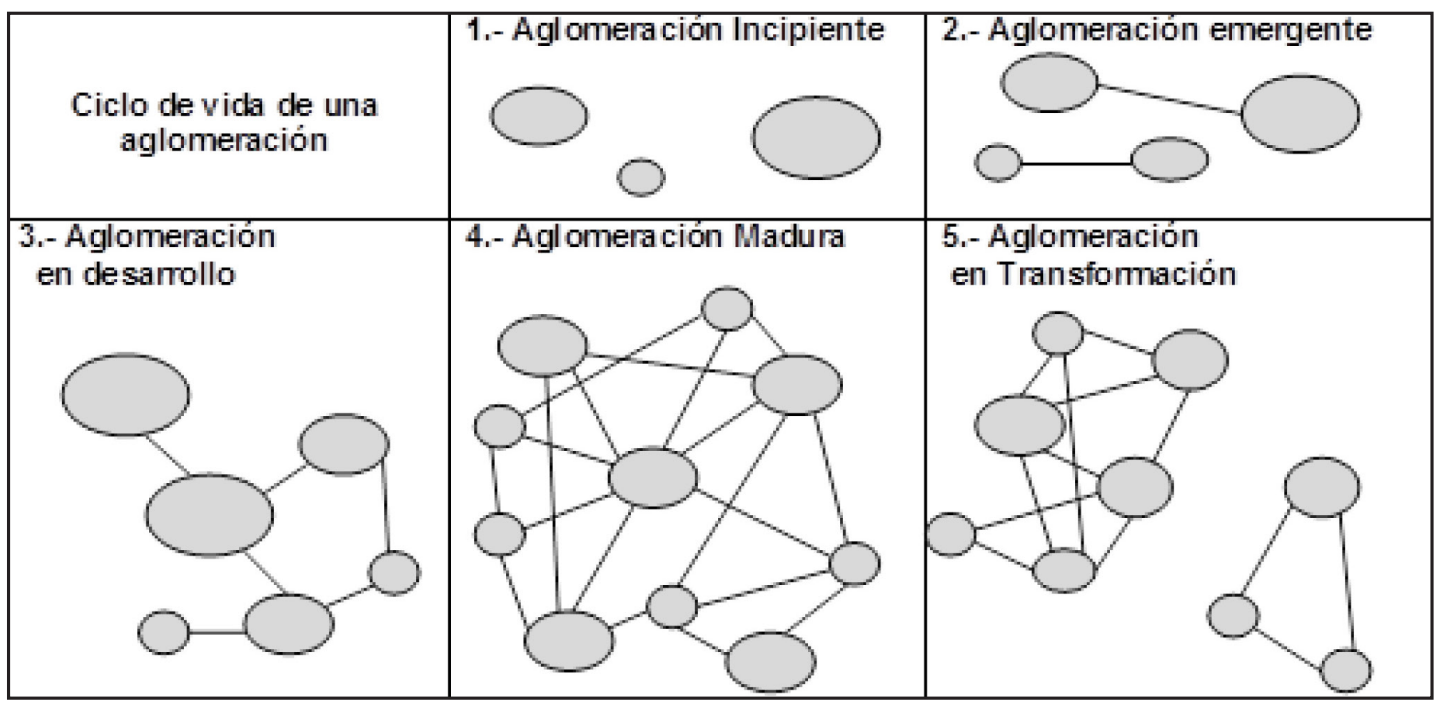

Fuente: Andersson et al. (2004). 
Es necesario precisar que, para el caso del turismo, la aglomeración (clusterización) se da en dos niveles, el primero referido a las actividades económicas interrelacionadas, pero no directamente vinculadas con el territorio, las denominadas actividades del sector. El segundo, involucra las actividades económicas referidas a espacios territoriales relativamente concentrados (regiones o localidades). En este sentido, la yuxtaposición de actividades sectoriales en un territorio especifico, se construye, desarrolla, consolida y transforma a partir de variables exógenas o endógenas, particulares e irrepetibles y mucho menos replicables en otro territorio. De ahí que la ubicación en un mismo espacio geográfico no garantiza por sí sola la integración de las empresas del sector turismo y de los sectores relacionados. Para tal propósito se deben generar las condiciones y el marco propicio para la participación tanto de empresarios como de líderes de los sectores público y social.

\section{EL CLÚSTER DE TURISMO}

La aplicación de esta metodología al turismo es una tarea reciente, incluso, la investigación de este enfoque aplicada al sector servicios, especialmente en el turismo médico, es un área nueva y por lo tanto poco desarrollada. De alguna manera, a este tipo de agrupaciones se les han aplicado los mismos principios que a los clústeres industriales.

A pesar de la amplia utilización de este modelo, no ha estado exento de críticas o precisiones respecto a su aplicación a la actividad turística. Miller y Gibson (2005), afirman que en su libro la ventaja competitiva de las naciones, Porter (1998), enfatiza que esta teoría aplica tanto para los servicios como para los bienes manufacturados. Sin embargo, solo en una frase de su texto se refiere de manera explícita al turismo. En lo subsecuente, en la prolífica literatura acerca del desarrollo económico basado en clústeres se hace mínima referencia al turismo, pero nunca Ilegaron a conceptualizarlo. En el mismo sentido, Navarro (2001) sostiene que, debido a su reciente aparición, en los análisis de este tipo de agrupaciones turísticas, con frecuencia se utiliza una terminología ambigua o contradictoria y para explicar su existencia se recurre a factores diferentes. No se toma en cuenta, de manera suficiente, la etapa del ciclo de vida en el que se encuentran, los sectores afectados o involucrados, o las características del espacio en el que se localizan. Además, en los estudios empíricos se emplean técnicas y métodos diferentes para su análisis, entre estas, las basadas en el análisis input-output, en la teoría de gráficos o mapeo, el análisis estadístico de correspondencia y el análisis de casos.

Otras posibles explicaciones para comprender estas desventajas del modelo de Porter aplicadas a los clústeres turísticos, son expuestas por Varisco (2007) quien afirma que pueden distinguirse tres enfoques que inciden en las propuestas para su fomento. El primero de ellos, se basa en la aplicación del concepto sin mayor conceptualización ni relación directa a la teoría que lo sustenta, por lo que sus propuestas no difieren mayormente de la planificación turística tradicional y se orientan a las manifestaciones económicas del turismo, y recaen principalmente en el sector público. El segundo enfoque, se centra en la adaptación del diamante de la competitividad de Porter, en el cual el gobierno juega un papel complementario por lo que su participación se analiza a partir de las funciones que realiza el organismo público de turismo. En el tercer enfoque, supone considerar la competitividad del destino turístico como una condición necesaria pero no suficiente para generar desarrollo. Implica, además, analizar un amplio conjunto de interrelaciones más allá del ámbito meramente económico, y reconocer la influencia de factores sociales, culturales y políticos en la dinámica productiva. 
El análisis realizado por Motoyama (2008), concluye que la naturaleza descriptiva y estática de esta teoría requiere de un análisis dinámico. Que exponga no únicamente como un grupo se organiza con éxito el día de hoy, sino que también explique cómo surgió, que tipo de desafíos tuvo que enfrentar y vencer, y quien jugó el importante papel de catalizador al interior del clúster. Por lo que el análisis debe ser por cada caso específico, longitudinal y profundo. Asimismo, señala que, si bien la integración fue uno de los argumentos centrales de esta teoría, ha demostrado ser débil al explicar cómo se desarrolla el aspecto de interconexión o establecimiento de redes dentro de una región. También sostiene que, reconociendo que las externalidades y la difusión del conocimiento son fundamentales para el desarrollo de una región, todavía se sabe poco de cómo sucede con exactitud esa transferencia. Argumenta que, a pesar de estas deficiencias, el modelo de Porter ha logrado tanta popularidad en su aplicación debido a tres razones principales: 1) Provee una explicación fácil, 2) Una dirección clara, y 3) Una justificación política para la intervención del gobierno. Pero que, en realidad, este método no proporciona casi ninguna guía de cómo desarrollar un clúster exitoso.

Otras críticas al modelo de Porter, se basan en no considerar dos variables exógenas de vital importancia en la competitividad de los clústeres y, sobre todo, de los aplicados a la actividad turística en los países en vías de desarrollo. De acuerdo con Kim y Wicks (2010) el modelo presenta vacíos importantes, entre los que se encuentran la contribución de las compañías multinacionales o transnacionales a las actividades económicas y las ventajas competitivas que aportan, así como el papel que desempeñan esas empresas en la integración de redes entren los participantes locales. En este sentido, indican que las empresas transnacionales pueden contribuir a la difusión del conocimiento en las empresas nacionales impulsando la competencia entre ambas, lo que a su vez mejoraría la competitividad del destino. Para lograr este efecto, es necesario conseguir que las empresas transnacionales se co-localicen a nivel local en lugar de permanecer aisladas.

Quienes apoyan la aplicación de este modelo a la actividad turística, consideran que el desarrollo del turismo es esencial para una región, porque el turismo puede crear empleos, estimular la inversión en infraestructura, provee recursos a los gobiernos por medio del pago de impuestos y el cobro de otros derechos; por lo tanto, contribuye de manera importante al Producto Interno Bruto (PIB) nacional e internacional (WHELAN; SUNDBLAND; INMAN, 1998). En este sentido, es necesario precisar que los productos turísticos son muy intensivos en la utilización de servicios, que requieren una colaboración muy estrecha entre los distintos proveedores ya que, en conjunto, todos ofrecen la experiencia de vacaciones (WEIERMAIR; STEINHAUSER, 2003).

Los productos turísticos son fundamentales para el proceso de integración de un clúster en una región. Se caracterizan por incorporar el patrimonio histórico, cultural y natural de la localidad, así como toda la gama de servicios que ofrecen las empresas del sector que permiten el arribo, permanencia y desplazamiento de los turistas (hospedaje, alimentación, transportación etc.), y los servicios de infraestructura que permiten la operación de toda la estructura turística.

Para identificar como se genera y distribuye el valor entre las distintas unidades productivas relacionadas con el turismo, generalmente de recurre al análisis de Cadena de Valor (Value Chain), la cual describe al conjunto de actividades que son requeridas para ofrecer un producto o servicio desde la simple idea, pasando por la fases de producción (que implica una combinación de la transformación física y la participación de varios productores de servicios) hasta la entrega a los consumidores finales (KAPLINSKY; MORRIS, 2002). 
Figura 4 - Clúster e integración de la cadena de valor

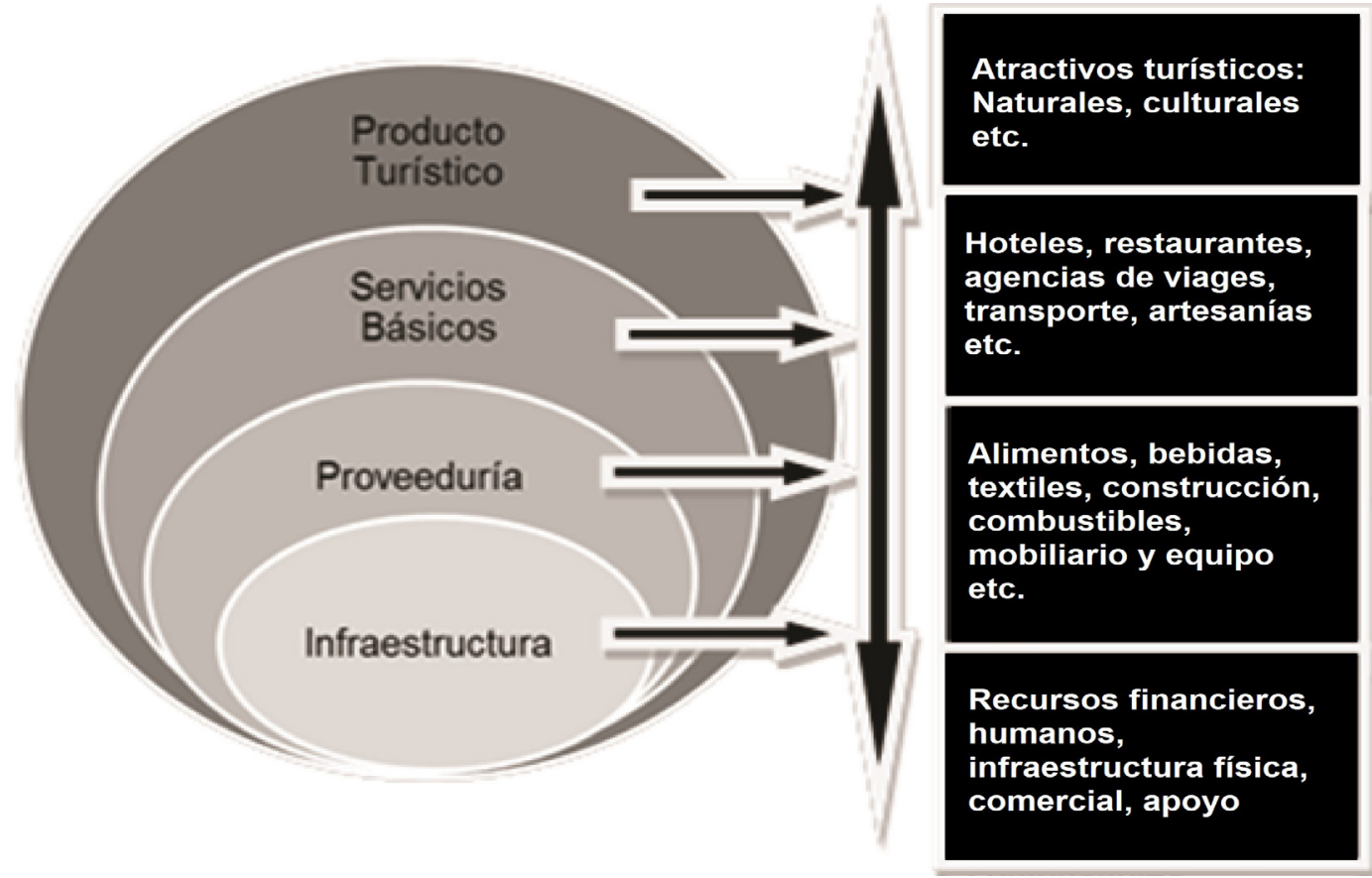

Fuente: Figura adaptada con base en el esquema de SECTUR (2007).

A pesar de que el análisis de cadena de valor se ha utilizado más para la producción de bienes, también es cierto que es aplicable al turismo, que si bien es cierto no ofrece bienes, también lo es el hecho de que es un complejo conjunto de servicios que se complementan con otros bienes para producirlo. Es necesario precisar que la mayoría de los servicios se caracterizan por la simultaneidad de la producción y el consumo, ya que no pueden ser almacenados. En el turismo, la producción y el consumo tienen lugar en una zona geográfica específica, el denominado destino turístico (VENTURA, 2011).

En este sentido Varisco (2004), identifica algunos de los factores principales de todo clúster de turismo: Los recursos naturales y culturales, que permiten identificar el potencial de atractividad de un destino. Los turistas, quienes conforman la demanda y generan el gasto y la derrama económica mediante el consumo de bienes y servicios. Los residentes locales, vinculados directa o indirectamente con el sector, quienes se ven beneficiados, y representan el propósito de todo programa o estrategia que se oriente a mejorar la calidad de vida. Además, el territorio, que debe ser dotado de la infraestructura necesaria para garantizar los niveles requeridos para la operación de las empresas y organizaciones turísticas. El diseño de los espacios urbanos, que permitirá reducir la competencia por el territorio entre el turismo y otras actividades económicas.

Por otro lado, autores como Jackson y Murphy (2002) argumentan que la aplicación del concepto de clúster de turismo es bastante apropiada, ya que el producto interactúa con las bases locales, promoviendo la realización de acciones conjuntas de las empresas que se relacionan entre sí, lo que da lugar al asociacionismo y aglomeración. Para estos autores, el desarrollo de un clúster debería contribuir a la transformación de las ventajas comparativas en ventajas competitivas. Brown y Geddes (2007) consideran como necesaria la participación de otros actores, no solo la simple aglomeración de proveedores para desarrollar un clúster exitoso. La intervención del gobierno resulta fundamental y debe orientarse a fomentar, financiar y desplegar estrategias para 
atraer la inversión privada y la inversión en infraestructura, ya que el turismo puede coadyuvar a superar las crisis económicas que se presentan a nivel regional y local.

Otro factor que se ha marginado en el análisis, pero que juega un papel de vital importancia por su participación en el clúster turístico, es el que se refiere a los conocimientos, habilidades y destrezas que deben o deberían tener los responsables de dichos agrupamientos, a quienes se les ha denominado de distintas formas: presidentes, directores, coordinadores, administradores, gerentes, entre otros. En ese sentido es importante incorporar los resultados del estudio realizado con el propósito de evaluar el nivel de calificación de los administradores de clústeres en Europa. Por un lado, participaron los administradores de clústeres, y por el otro, por participantes y miembros de dichos clústeres. El estudio se realizó en el marco de la Inno-Net, proyecto ECOCluster Network. Establecida por la Comisión Europea DG, empresa e industria. Se incluyeron clústeres del sector automotriz, de negocios y servicios financieros, de agricultura, de caza y pesca, de textiles, ropa y calzado deportivo, así como de turismo de bienestar y turismo de salud.

Una primera afirmación al respecto, señala que, como el puesto de administrador de clúster es una profesión relativamente nueva, para la que todavía no se ha definido un perfil profesional específicamente para desarrollar esta función, se espera que un administrador tenga un profundo conocimiento sobre la actividad específica del clúster, conocimientos sobre el tipo de negocios que se desarrolla, así como conocimientos técnicos de la forma en que se desarrollan las actividades.

Entre las habilidades que deben poseer destacan: la capacidad de gestión, para la comunicación, el uso de las tecnologías de la información y comunicación, manejo aceptable de otros idiomas, experiencia y habilidades para identificar las políticas inherentes y relevantes en su área, así como para identificar e interactuar con quienes definen las políticas públicas de interés especial para el mismo. Además de otras características como liderazgo de calidad para llegar a compromisos claros y, por supuesto, los administradores deben ser flexibles para adaptar continuamente los servicios que se ofrecen a los integrantes, socios o miembros, o mejor aún, para desarrollar nuevos servicios (PRO INNO EUROPE, 2009).

Al referirse específicamente a los 13 clústeres de turismo médico incluidos en el estudio (del total de 107 administradores de clústeres que participaron en la investigación, 13 correspondieron a los de turismo de salud o de turismo médico), se encontró que, entre más nueva es la aglomeración mayor es la importancia que adquiere el desarrollo de una estrategia. De los conformados entre 1991 y 1998, el 53\% considera esta actividad sumamente importante, mientras que entre los conformados entre el 2004 y el 2008, el porcentaje alcanza el 82\%.

Respecto a la importancia de la conformación de redes y el lobbying, la relación se da en sentido inverso, esto es, el 68\% de los clústeres más nuevos, conformados entre el 2004 y el 2008, otorgan una alta prioridad a estas actividades, mientras que de los conformados entre 1991 y 1998, el 80\% considera sumamente importante realizar estas actividades.

Al indagar acerca del perfil socio demográfico de los administradores, se encontró que siete tiene formación profesional en el área de administración y negocios, derecho o ciencias políticas, tres en ingeniería o tecnologías de la información, y los tres restantes indicaron otras áreas de formación. Al analizar la distribución por género se encontró que diez son administrados por mujeres y únicamente tres por hombres. Al referirse al número de actividades relacionadas con la internacionalización que desarrollan y que son de vital importancia para el posicionamiento actual del clúster, siete administradores indicaron que desarrollan entre uno y cuatro tareas orientadas a este fin, los restantes seis no señalaron llevar a cabo este tipo de tareas. 
En suma, a pesar de las críticas, opiniones desfavorables y debates, el diamante de la competitividad de Porter puede ser un instrumento útil para la comprensión y gestión de la actividad turística a nivel local, con las adecuaciones en su conceptualización y la actualización de los actores que lo configuran. Domínguez (2001) sugiere que, a los componentes del diamante de la competitividad, deben agregarse los siguientes elementos para acercarlo más a su aplicación al turismo:

A las condiciones de la oferta (o condiciones de los factores productivos) que incluyen los recursos humanos, físicos, de capitales, de conocimiento e infraestructuras, deben añadirse los recursos culturales o históricos y la superestructura del turismo.

Respecto a las condiciones de la demanda, señala que debe considerarse de capital importancia la presencia in situ de consumidores con altos niveles de exigencia, de diversificación y de dinamismo, ya que estas características son un elemento indispensable en los procesos de innovación y, simultáneamente, son causa y efecto en las necesidades de formación y profesionalización de los prestadores de servicios turísticos, estimulando el perfeccionamiento de productos y mejorando los servicios ofrecidos, lo que trae como consecuencia la eficiencia empresarial.

Al referirse a los sectores de apoyo y relacionados, indica que las relaciones que mantengan las empresas turísticas con sus proveedores y con los operadores que controlan la demanda turística final, son fundamentales a la hora de detectar el nivel de complejidad estructural del destino. Debe ponerse especial atención al grado de dependencia de las compañías touroperadoras y los efectos de arrastre que el turismo produce en otros sectores económicos conexos.

Por su parte, la estrategia, estructura y rivalidad implicarían las relaciones entre las empresas turísticas y el entorno social ya que constituyen un punto clave en la formación, evolución y expansión de los clústeres. Los destinos turísticos convencionales presentan una profunda atomización de las empresas turísticas con las que compiten, particularmente de las grandes cadenas que ofrecen todos los servicios incluidos.

Existen otros dos factores que influyen de manera directa en la competitividad, el primero de ellos es el gobierno, quien debe propiciar una política turística encaminada hacia la consecución de un entorno en que las empresas puedan mejorar sus ventajas competitivas. No debe ponerse demasiado énfasis en las variables macroeconómicas que a la larga podrían debilitar la ventaja competitiva, por el contrario, deben orientarse más al control de variables en el nivel microeconómico, aunque coordinada tanto a nivel local como regional, e incluso a nivel nacional. Habría que agregar la casualidad que puede crear discontinuidades y brindar oportunidades para la sustitución de empresas en diferentes países. Entre las casualidades más recurrentes, se encuentran los desastres naturales y las decisiones del entorno político que pueden afectar los flujos turísticos (DOMíNGUEZ, 2001).

\section{EL CLÚSTER DE TURISMO MÉDICO}

Es necesario establecer que se entiende por un clúster de turismo médico, el cual podría definirse como un complejo entramado de servicios médicos (Hospitales, clínicas e institutos) que desempeñan el papel de atractivo principal en el destino, que se combinan con facilidades y servicios turísticos proporcionados por empresas privadas de manera complementaria (Hoteles, restaurantes, transporte local, agencias de viaje, y todos aquellos servicios adicionales, diseñados específicamente para este segmento de viajeros), todos coordinados por un representante 
gubernamental, el cual proveerá los servicios públicos, infraestructura y apoyos para la inversión privada en este sector.

En la realidad, elegir un destino por razones de turismo médico implica considerar todavía más factores adicionales que para la elección de un destino para vacaciones tradicionales. Entre los criterios más relevantes se encuentran la calidad del sistema de salud y la imagen de las instalaciones sanitarias del destino. Así como los recursos disponibles tanto en personal como en instalaciones y facilidades médicas. Los precios de los procedimientos o tratamientos y servicios relacionados, el precio de las medicinas y, por último, la atractividad turística del destino (KESAR; RIMAK, 2011).

Recientes esfuerzos para impulsar el turismo médico, han obligado a precisar su conceptualización como punto de partida (AIT \& B2LS, 2008), ahora, la atención se centra en la medicina y los tratamientos, así como en las cirugías y procedimientos que se ofrecen en hospitales y centros médicos, y la participación del sector turismo se considera como secundaria, y se requiere su incorporación para realizar tres acciones esenciales:

a) Facilitar todos los trámites de viaje, junto con todos los servicios pertinentes desde y hacia los destinos de procedencia.

b) Ofrecer actividades de ocio, recreativas, culturales o de entretenimiento que se le permita realizar al paciente, como parte de su programa médico.

c) Realizar actividades de promoción, distribución y comercialización de los servicios médicos.

En suma, los esfuerzos de conjuntar a los sectores médicos y de turismo para atender a un nicho emergente de consumidores requieren necesariamente, comprender cuales serían las nuevas dinámicas que deben empezar a atender las empresas pertenecientes a ambos sectores se pueden sintetizar en los siguientes aspectos, planteados por Toledo, Marroquín y Castroman (2004) en su modelo de sistema interfuncional interrelacionado de la competitividad del destino turístico (SIIC). Que si bien es cierto se refiere únicamente a los clústeres de turismo, también lo es el hecho de que refleja con toda claridad el enfoque que precisan los clústeres de turismo médico, para enfrentar los retos que les impone la dinámica globalizada en la que se desarrollan.

Con base en lo anterior, se establece que la oferta debe estar dirigida a segmentos o nichos específicos, integrada con productos planificados para poder satisfacer las necesidades de visitantes y turistas; por lo tanto, el ciclo de la actividad turística está en función de la integración y diversificación de productos, debido a que la demanda se caracteriza por ser altamente calificada y experimentada, distinguiéndose por consumidores muy exigentes y sofisticados en sus motivaciones y necesidades.

Debe establecer mecanismos y condiciones para que la elaboración, uso y distribución de la tecnología sea compartida por todos los agentes, enfatizando la orientación al cliente.

Para permitir la entrada y salida de competidores, debe contar con un marco legal detallado que motive e incentive las inversiones nacionales y extranjeras, que le permitan articularse a nivel global. Pero al mismo tiempo, la regulación debe ser altamente planificada y detallada para garantizar la sustentabilidad del clúster, a través de incentivar las certificaciones ecológicas y de sustentabilidad. De ahí que resulte indispensable la obligatoriedad de los miembros para establecer relaciones con los sectores público, privado y la sociedad civil, con el propósito de establecer un enfoque de planificación y gestión intersectorial, basada en metodologías, técnicas y técnicos específicos que propicien la integración y cooperación vertical y horizontal, que den como resultado un plan estratégico con visión de largo plazo, tanto para el clúster en su conjunto 
como para las empresas en lo individual, con objetivos orientados a la competitividad local pero de alcance global.

Para la consecución de dichas metas, la definición del marco legal y de las políticas de acción tanto del gobierno como de las empresas, resulta de capital importancia ya que ambas deben alinearse con los objetivos del plan estratégico que se haya establecido.

Respecto a la estrategia competitiva, señala que debe ser fuerte, constructiva y capaz de generar alianzas estratégicas a escala regional y global; mientras que en la estrategia cooperativa debe incluir la participación del sector público, la iniciativa privada y la sociedad civil, y debe estar integrada para atender las necesidades y motivaciones del usuario. Establece también, que la estrategia relacional con los turistas debe estar integrada al clúster, planificada para cada agente y sociedad local.

Además, la operatividad cotidiana precisa que las tareas de investigación y desarrollo, las actividades de ejecución del plan estratégico, así como el programa de educación, capacitación y cultura empresarial, se desarrollen de manera conjunta por parte de los directivos, pero también de manera individual en cada una de sus empresas afiliadas. Sólo de esta manera, se podrá dar cumplimiento a las metas del plan estratégico.

Se recomienda que las actividades de evaluación y retroalimentación, se realicen de manera formal, sistematizada, detallada y con carácter de obligatorias, pero con la colaboración de consultores externos, ya que de esta manera se pueden obtener resultados objetivos, y evitar conflictos internos entre los participantes.

Con el fin de llevar a cabo las actividades de promoción, ya sea a nivel regional nacional o internacional, además de utilizar las instancias propias ya establecidas o preexistentes, tales como embajadas, consulados, etc., también se deben crear agencias o instancias propias que realicen esta actividad. Garantizando la estandarización de la información que se pretenda difundir, para evitar crear confusiones o desgastar la imagen del clúster.

Por último, se debe diseñar la imagen corporativa que permita posicionarlo en su conjunto, dicha imagen debe representar la marca. Por lo tanto, debe expresar la función principal que se desarrolla; debe ser reconocible y diferenciable de otras marcas que se ubican en el mismo ámbito de negocios en que se desenvuelve, y mantenerse a nivel local, regional, nacional e internacional.

En síntesis, los retos de turismo médico son similares a los retos que enfrenta la actividad turística en general. Convertir las ventajas comparativas en ventajas competitivas y sustentables, requieren no solamente un marco político coherente y un sector privado dinámico, sino también de instituciones eficientes para traducir las medidas políticas en programas, capaces de lograr la integración en las redes de turismo mundial y, sobre todo, llevar a cabo las acciones concretas que permitan establecer vínculos con otros sectores de la economía y a lo largo de todas las cadenas de valor. Estos son los elementos clave para su desarrollo y consolidación (UNITED NATIONS STEERING COMMITTEE ON TOURISM FOR DEVELOPMENT [UNDP], 2011).

\section{CONCLUSIONES}

Con base en el análisis desarrollado a lo largo de este artículo, se puede señalar que la conceptualización deficiente o parcial del fenómeno del clúster de turismo médico que existe, propicia la carencia de sistemas, métodos e instrumentos propios que permitan su conocimiento y comprensión integral; lo que, a su vez, impacta en los niveles de competitividad de los actores locales que lo conforman, debido a la falta de indicadores específicos para su seguimiento y 
evaluación. El actual enfoque de clúster, no es el más apropiado para la actividad turística, ya que se retoman características del sector industrial y se aplican al sector turismo. Para una actividad en ciernes, como el turismo médico, quedan pendientes varios aspectos para trabajarse en futuras líneas de investigación, entre las que se encuentran evaluar su impacto en la captación de divisas, en la generación de empleos y su contribución en la mejora de la calidad de vida de los habitantes de los países exportadores.

Para una mejor comprensión del fenómeno, es conveniente realizar esfuerzos académicos por conceptualizar esta actividad, a partir de una nueva perspectiva desde y para el turismo, tomando como base las características de producción, comercialización, consumo, evaluación y mejoramiento de los productos turísticos. Como resultado de la revisión documental respecto a la definición, uso y aplicación del modelo de clúster, se encontró que existen discrepancias respecto a la pertinencia de la aplicación del modelo para el estudio y gestión del turismo. No se puede rechazar categóricamente su utilidad para tal propósito, pero tampoco puede ser aceptada sin mayores cuestionamientos. En este sentido, hay que reconocer que su aplicación actual carece de una conceptualización específica desde y para el desde turismo. Tal parece que de su aplicación a las actividades industriales se transfirió directamente a las actividades del turismo, sin mayor justificación que la concentración de firmas o empresas en espacios locales y que, por lo tanto, la cooperación está determinada por la proximidad geográfica, pasando por alto una diferencia fundamental: la presencia del turista en el espacio local de producción y consumo de productos turísticos y las repercusiones que esto genera en los procesos de recepción, atención y regreso del visitante a su lugar de residencia.

Debido a que los espacios dedicados a la fabricación o ensamble industrial de bienes se han diseñado para satisfacer las necesidades de los productores, sus características urbanas se orientan, principalmente, a satisfacer las necesidades de movilizar a la fuerza laboral, al transporte de materias primas y de productos terminados, así como a la disponibilidad de infraestructura que facilite su importación y exportación; no requiere necesariamente un ambiente urbano cuidado, funcional y urbanísticamente diseñado para el consumidor final ya que este se encuentra a cientos o miles de kilómetros del centro de producción, y la mercancía será transportada hacia su lugar de residencia.

Por su parte, en el turismo esta dinámica se revierte y cambia el sentido y requerimientos del diseño del entorno urbano que es, a la vez, el centro de producción y consumo. Aquí, por el contrario, el visitante se traslada desde su lugar de residencia habitual para consumir in situ los productos turísticos; como el diseño del espacio turístico debe responder a necesidades, gustos preferencias cambiantes por parte de la demanda, las prácticas al interior de los sitios turísticos suelen ser menos estáticas e invariables de lo que generalmente se supone. Casi todos los destinos que deseen atraer a un nuevo segmento o nicho de visitantes, como es el caso del turismo médico, se ven obligados a modificar el espacio urbano lo que implica, además de la inversión de las empresas privadas en instalaciones médicas, inversiones del gobierno en instalaciones públicas para facilitar su llegada, permanencia y regreso a su destino; esto incluye cuidar los aspectos de funcionalidad, apariencia física y características arquitectónicas desde los puntos de acceso (aeropuertos, puertos, carreteras, puertos de cruces fronterizos), pasando por la limpieza de la ciudad, la seguridad en el destino, la disponibilidad de información en su idioma, hasta la señalización turística para orientarlo y facilitar su experiencia. Además, los destinos turísticos deben incluir el cumplimiento de estándares en los ámbitos de la sustentabilidad y el 
respeto a la cultura local, no solamente por necesidades de la oferta, sino particularmente por los requerimientos y necesidades de la demanda.

En suma, si se aceptan las limitaciones del enfoque de Porter para su aplicación al turismo, y se admite su utilidad solo como la base teórica inicial pero que requiere actualizarse, enriquecerse y ampliarse; debe asumirse entonces que aplicar este enfoque a la actividad turística representa desafíos conceptuales significativos, o por lo menos que se requiere de una perspectiva diferente a la manera que se aplica hasta hoy en el sector industrial. El nuevo paradigma debe orientarse a explicar cómo la producción, exportación y consumo de los productos se realiza simultáneamente en el mismo espacio, con la interacción sincrónica de oferentes y demandantes de productos turísticos y médicos, incorporando la participación de las trasnacionales y su intervención en los procesos de integración y difusión del conocimiento de manera dinámica. Solo bajo estas circunstancias, y considerando el dinamismo del turismo como una constante, el modelo de clúster sería válido para el turismo médico.

\section{REFERENCIAS}

ANDERSSON, T.; SCHWAAG-SERGER, S.; SÖRVIK, J.; WISE, E. The cluster policy whitebook. [S.I.]: International Organization for Knowledge Economy and Enterprise Development (IKED), 2004.

AIT \& B2LS. Medical tourism development strategy. El Cairo, Egypt: Federation of Egyptian industries. Final Report, PS-401. 2008. Disponible en: https://www.yumpu.com/en/document/read/10719004/medicaltourism-development-strategy-imc-egypt. Acceso en: 30 jun. 2020.

BROWN, R. Cluster dynamics in theory and practice with application to Scotland. Regional and Industrial Policy Research Paper, Glasgow, England, n. 38, 2000.

BROWN, K.; GEDDES, R. Resorts, culture and music: the Cape Breton tourism cluster. Tourism Economics, [S.I.], v. 13, n. 1, p. 129-41, 2007.

CASTILLO, M.; LOZANO, M. Apuntes para la investigación turística. Cuerpo Académico de Turismo. México: Universidad de Quintana Roo, 2006.

DAHL, M. S. What is the essence of geographic clustering. Aalborg, Denmark: DRUID Nelson \& Winter Conference, 2001.

DOMÍNGUEZ, M. La creación de clústeres turísticos como instrumento para la mejora competitiva de los destinos: una aplicación a las Rías Bajas Gallegas. Investigaciones Europeas de Dirección y Economía de la Empresa, [S.I.], v. 7, n. 3, p. 119-38, 2001.

IRE. Regional clustering and networking as innovation drivers. Design of cluster initiatives -an overview of policies and praxis in Europe. Learning module 1. 2001. Disponible en: http://www.clusterpolisees3. eu/ClusterpoliSEEPortal/resources/cms/documents/2012.09.11_Regional_clustering_and_networking_ as_innovation_drivers.pdf. Acceso en: 30 jun. 2020.

JACKSON, J.; MURPHY, P. Tourism destinations as clusters: analytical experiences from the new world. Tourism and Hospitality Research, [S.I.], v. 4, n. 1, 2002.

KAPLINSKY, R.; MORRIS, M. A handbook for value chain research (Manual preparado para IDRC). 2002. Disponible en: http://www.fao.org/fileadmin/user_upload/fisheries/docs/Value_Chain_Handbool.pdf. Acceso en: 30 jun. 2020. 
KESAR, O.; RIMAK, K. Medical tourism development in Croatia. Zagreb International Review of Economics \& Business, Zagreb, Croatia, v. 14, n. 2, p. 107-34, 2011.

KETELS, C. H.; LINDKVIST, G.; SÖLVEL, Ö. Cluster initiatives in developing and transition economies. Stockholm, Sweden: Center for Strategy and Competitiveness, 2006.

KIM, N.; WICKS, B. Rethinking tourism cluster development models for global competitiveness. International CHRIE Conference-Refereed Track. Paper 28. Amherst, U.S.A.: University of Massachusetts, 2010.

MILLER, M.; GIBSON, J. Cluster-based development in the tourism industry: putting practice into theory. Applied Research in Economic Development, p. 47-64, 2005.

MOTOYAMA, Y. What was new about the cluster theory? What could it answer and what could it not answer. Economic Development Quarterly, v. 22, n. 4, 2008. Disponible en: https://journals.sagepub.com/ doi/10.1177/0891242408324373. Acceso en: 30 jun. 2020.

NAVARRO, A. M. Análisis y políticas de clústeres: teoría y realidad. Ekonomiaz, Madrid, España, n. 53, 2003.

NAVARRO, A. M. El análisis y la política de clústeres. Documentos de Trabajo del Instituto de Análisis Industrial y Financiero, Madrid, España, n. 27, 2001.

PORTER, M. Clusters of innovation: Regional Foundations of U.S. Competitiveness. Washington, DC, U.S.A.: Council on Competitiveness, 2001.

PORTER, M. Clusters and the new economics on competition. Harvard Business Review, Harvard, U.S.A., Reprint 98609, p. 77-90, nov./dez. 1998.

PRO INNO EUROPE. CMQ: Cluster Manager Qualification. Results of a comprehensive survey on tasks, skills \& training needs of European cluster managers. 2009. Disponible en: http://docplayer.net/9359886Cmq-cluster-manager-qualification.html. Acceso en: 30 jun. 2020.

SECTUR. Integración de agrupamientos turísticos competitivos, "Clusters turísticos". Programa de Competitividad. Serie de documentos técnicos. Secretaría de Turismo, México. 2007. Disponible en: https://docplayer.es/66353418-Integracion-de-agrupamientos-turisticos-competitivos-clusters-turisticos. html. Acceso en: 21 ago. 2019.

TELLO, M. Desarrollo económico local, descentralización y clústers: teoría, evidencia y aplicaciones. Lima, Perú: CENTRUM Católica- Centro de Negocios de la Pontificia Universidad Católica del Perú, 2008.

TOLEDO, G.; MARROQUÍN, E.; CASTROMAN, Á. Estrategia internacional de un clúster turístico latinoamericano. Estudio de caso, Sauipe, Brasil. 2004. Disponible en: https://bibliografiaturism.wixsite. com/bibliotecadeturismo/single-post/2018/02/07/ ESTRATEGIA-INTERNACIONAL-DE-UN-CLUSTERTUR\%C3\%8DSTICO-LATINOAMERICANO-ESTUDIO-DE-CASO-SAUIPE-BRASIL. Acceso en: 18 feb. 2020.

TURNER, L. First world health care at third world prices: Globalization, bioethics and medical tourism. BioSocieties, London, v. 2, n. 3, p. 303-25, 2007.

UNITED NATIONS STEERING COMMITTEE ON TOURISM FOR DEVELOPMENT (UNDP). Tourism and poverty reduction strategies in the integrated framework for least developed countries. 2011. Disponible en: https://www.undp.org/content/undp/en/home/librarypage/poverty-reduction/trade_content/tourismand-LDCs.html. Acceso en: 30 jun. 2020.

VARISCO, C. Iniciativas de desarrollo local y fomento del clúster turístico. Aportes y Transferencias, Mar del Plata, Argentina, v. 11, n. 1, 2007. 
VARISCO, C. El clúster turístico de Miramar. Aportes y Transferencias, Mar del Plata, Argentina, v. 8, n. 2, p. 61-88, 2004.

VENTURA, D. V. El turismo, su cadena productiva, y el desarrollo incluyente en América Latina: los casos de Brasil y México. Serie Comercio y crecimiento inclusivo (138), 1- 81. 2011.

WEIERMAIR, K.; STEINHAUSER, C. New tourism clusters in the field of sport and health: the case of Alpaine Wellness. $12^{\text {th }}$ International Tourism Symposium. Barcelona, España. 2003.

WHELAN, J.; SUNDBLAND, L.; INMAN, C. Diagnostic: training needs assessment of the tourism sector in Central America. INCAE, 1998. Disponible en: http://x.incae.edu/EN/clacds/publicaciones/pdf/cen601.pdf

\section{Sobre los autores:}

Jorge Carlos Morgan Medina: Doctor en Planificación Territorial y Desarrollo Regional por la Universidad de Barcelona, España; maestro en Gestión Pública del Turismo, Sostenibilidad y Competitividad, por la Universidad Internacional de Andalucía, España; posgraduado en Planificación y Gestión del Espacio Turístico, por la Universidad de Alicante, España; posgraduado en Desarrollo Humano Sostenible, por la Universidad Central Europea, Hungría; diploma de Estudios Avanzados por la Universidad de Barcelona, España; y graduado en Turismo, por la Universidad Autónoma de Baja California (UABC), México. Profesor-investigador de Tiempo Completo en la Facultad de Turismo y Mercadotecnia de la UABC, México; y profesor adjunto de Posgrado del Centro de Estudios Universitarios Xochicalco, México; con 22 años de experiencia docente en Licenciatura, Especialidad, Maestría y Doctorado. E-mail: jorgemorgan@uabc.edu.mx, Orcid: http://orcid.org/0000-0003-3536-9986

Onésimo Cuamea Velázquez: Doctor en Estudios del Desarrollo Global por la Universidad Autónoma de Baja California (UABC), México; profesor-investigador de Tiempo Completo en la Facultad de Turismo y Mercadotecnia de la UABC, México. Presidente de la Asociación Mexicana de Centros de Enseñanza Superior en Turismo y Gastronomía (AMESTUR). E-mail: onesimo@uabc.edu.mx, Orcid: http://orcid.org/0000-0002-5568-9883

Ario Refugio Estrada Gaxiola: Doctor en Ciencias Administrativas por la Universidad Autónoma de Baja California (UABC), México; profesor-investigador de Tiempo Completo en la Facultad de Turismo y Mercadotecnia de la UABC. Evaluador acreditado (RCEA) del Sistema Nacional de Evaluación Científica y Tecnológica (CONACYT, México), como especialista del Sector de Turismo. E-mail: ario@uabc.edu.mx, Orcid: http://orcid.org/0000-0003-0603-0326 
